manifold. Thus a cartesian factor of a homotopy manifold is not necessarily a homotopy manifold.

\title{
REFERENCES
}

1. R. Bing, $A$ decomposition of $E^{3}$ into points and tame arcs such that the decomposition space is topologically different from $E^{3}$, Ann. of Math. vol. 65 (1957).

2. M. Curtis and R. Wilder, The existence of certain types of manifolds, Trans. Amer. Math. Soc. vol. 91 (1959) pp. 152-160.

3. H. Griffiths, $A$ contribution to the theory of manifolds, Michigan Math. J. vol. 2 (1953) pp. 61-89.

4. R. Roxen, $E^{4}$ is the Cartesian product of a totally non-euclidean space and $E^{1}$, Notices Amer. Math. Soc., Abstr. 563-1, vol. 6 (1959) p. 641.

5. S. Smale, A Vietoris mapping theorem for homotopy, Proc. Amer. Math. Soc. vol. 8 (1957) pp. 604-610.

6. R. Wilder, Monotone mappings of manifolds, Pacific J. Math. vol. 3 (1957) pp. 1519-1528.

7. - Monotone mappings of manifolds II, Michigan Math. J. vol. 5 (1958) pp. 19-25.

The Seoul National University, Seoul, Korea

\section{LOGARITHMICALLY EXACT DIFFERENTIAL FORMS}

\section{RICHARD S. PALAIS ${ }^{1}$}

Let $M$ be a connected, differentiable $\left(=C^{\infty}\right)$ manifold. Let $C^{1}(M, C)$ denote the complex vector space of complex valued one-forms on $M$ : an element $\omega$ of $C^{1}(M, C)$ is a function which assigns to each $x \in M$ a linear map $\omega_{x}$ of $M_{x}$ (the tangent space to $M$ at $x$, a real vector space) into the complex numbers $C$, such that if $X$ is a differentiable vector field on $M$ then $x \rightarrow \omega_{x}\left(X_{x}\right)$ is a differentiable complex valued function on $M$. Each element $\omega$ of $C^{1}(M, C)$ can be written uniquely in the form $\mu+i \nu$ where $\mu$ and $\nu$ are real valued one-forms on $M$, and we put $\mu=\operatorname{Re} \omega$ and $i \nu=\operatorname{Im} \omega$. We write $Z^{1}(M, C)$ for the subspace of $C^{1}(M, C)$ consisting of closed forms and $B^{1}(M, C)$ for the subspace of $Z^{1}(M, C)$ consisting of exact forms.

An element of $C^{1}(M, C)$ will be called logarithmically exact if it is of the form $d f / f$ for some nowhere vanishing, differentiable, complex valued function $f$ on $M$. Since $d(d f / f)=\left(f d^{2} f-d f \wedge d f\right) / f^{2}=0$ and $d f / f-d g / g=d(f / g) /(f / g)$ it is clear that the set $L^{1}(M, C)$ of logarithmically exact one-forms is a subgroup (but not in general a subspace)

Received by the editors February 24, 1960.

1 The author is a National Science Foundation postdoctoral fellow. 
of $Z^{1}(M, \mathbf{C})$ and, since $d g=d(\exp g) /(\exp g), B^{1}(M, \boldsymbol{C})$ is a subgroup of $L^{1}(M, \boldsymbol{C})$. The quotient group $L^{1}(M, \boldsymbol{C}) / B^{1}(M, \boldsymbol{C})$ is thus a subgroup of the complex vector space $H^{1}(M, \mathrm{C})=Z^{1}(M, \mathrm{C}) / B^{1}(M, \mathrm{C})$ (the one-dimensional complex deRham cohomology space of $M$ ) and so a torsion free abelian group which we will call the group of $\log a$ rithmic cohomology classes of $M .^{2}$

If $\omega$ is a logarithmically exact one-form on a differentiable manifold $N$ and $\Phi: M \rightarrow N$ is a differentiable map then $\delta \Phi^{*}(\omega)$ is a logarithmically exact one-form on $M$. In fact if $\omega=d f / f$ then $\delta \Phi^{*}(\omega)=d(f \circ \Phi) /(f \circ \Phi)$. Now if $I$ denotes the identity map of the unit circle into the complex plane then $\lambda=d I / I$ is a logarithmically exact one-form on the circle. If $\theta$ is a local determination of the argument function on the circle then $\lambda=d\left(e^{i \theta}\right) /\left(e^{i \theta}\right)=i d \theta$ so $\lambda$ is purely imaginary. A logarithmically exact one-form on $M$ will be called induced if it is of the form $\delta \Phi^{*}(\lambda)$ for some differentiable map $\Phi$ of $M$ into the unit circle. Clearly such a form is purely imaginary. Since $\delta \Phi^{*}(\lambda)=\delta \Phi^{*}(d I / I)=d(I \circ \Phi) /(I \circ \Phi)$ $=d \Phi / \Phi$ a logarithmically exact one-form is induced if and only if it can be written in the form $d f / f$ where $|f| \equiv 1$. Now if $f$ is a nowhere vanishing function on $M$ and then $|f|$ is differentiable, $g=f /|f|$ is differentiable, and $|g| \equiv 1$. Since $d f / f=d(|f| g) /(|f| g)=d|f| /|f|$ $+d g / g=d \log |f|+\delta g^{*}(\lambda)$ and since $d \log |f|$ is clearly real while as remarked above $\delta g^{*}(\lambda)$ is purely imaginary we see that: If $\omega$ is a logarithmically exact one-form on $M$ then $\operatorname{Re} \omega$ is exact and $\operatorname{Im} \omega$ is an induced logarithmically exact form. It follows in particular that real logarithmically exact forms are exact, imaginary logarithmically exact forms are induced, and that every logarithmic cohomology class contains an induced logarithmically exact one-form.

We shall now examine how logarithmically exact forms can be characterized by their periods. A path on $M$ is a piecewise differentiable map $\gamma$ of a closed interval $[a, b]$ into $M$ and is called a cycle if $\gamma(a)$ $=\gamma(b)$. If $\omega$ is a one-form on $M$ we write $\omega(\gamma)$ for the integral of $\omega$ over $\gamma$. If $\omega$ is closed and $\gamma$ a cycle then $\omega(\gamma)$ depends only on the cohomology class $[\omega]$ of $\omega$ in $H^{1}(M, \boldsymbol{C})$ and the integral homology class $[\gamma]$ of $\gamma$ in $H_{1}(M, Z)$ and we write it as $([\gamma],[\omega])$. The set of numbers of the form $([\gamma],[\omega])$ as $[\gamma]$ varies over $H_{1}(M, Z)$ is a sub-

2 The author was introduced to the notion of logarithmically exact forms in a conversation with Professor I. E. Segal. They arose naturally while Segal was investigating certain generalizations of quantum field theory that occur when Euclidean threespace is replaced by more general manifolds. In a certain sense $L^{1}(M, C) / B^{1}(M, C)$, the group of logarithmic cohomology classes, classifies the unitary equivalence classes of certain natural representations of the generalized "Heisenberg commutator relations." It was Segal's conjecture that this group had a rank equal to the first Betti number of $M$ (Theorem B) that motivated the author's work on this paper. 
group of $C$ called the group of periods of $[\omega]$ (or of $\omega$ ) and denoted by $\Omega([\omega]$ ) (or by $\Omega(\omega)$ ). We now come to our main result. Let $2 \pi i \boldsymbol{Z}$ denote the subgroup of $\boldsymbol{C}$ consisting of integral multiples of $2 \pi i$. Then

TheOREM A. A closed one-form $\omega$ on $M$ is logarithmically exact if and only if its group $\Omega(\omega)$ of periods is a subgroup of $2 \pi i \boldsymbol{Z}$. Equivalently a one-dimensional cohomology class of $M$ is logarithmic if and only if its group of periods is a subgroup of $2 \pi i \boldsymbol{Z}$.

Proof. If $\omega$ is logarithmically exact then by a previous remark it is cohomologous to an induced logarithmically exact form $\delta \Phi^{*}(\lambda)$. If $\gamma$ is a cycle in $M$ then $\Phi \circ \gamma$ is a cycle in the circle which is then an integral multiple $n \gamma_{0}$ of the fundamental cycle $\gamma_{0}$ of the circle. Then $\omega(\gamma)=\delta \Phi^{*}(\lambda)(\gamma)=\lambda(\Phi \circ \gamma)=n \lambda\left(\gamma_{0}\right)$. Since $\lambda\left(\gamma_{0}\right)=\int_{\theta=0}^{\theta=2 \pi} d\left(e^{i \theta}\right) / e^{i \theta}=2 \pi i$ it follows that $\Omega(\omega) \subseteq 2 \pi i \boldsymbol{Z}$. Conversely suppose $\Omega(\omega) \subseteq 2 \pi i \boldsymbol{Z}$. Let $p_{0}$ be a fixed point of $M$ and define a complex valued function $f$ on $M$ by $f(p)=\exp (\omega(\gamma))$ where $\gamma$ is a path joining $p_{0}$ to $p$. If $\gamma_{1}$ and $\gamma_{2}$ are two paths joining $p_{0}$ to $p$ then $\gamma_{1}-\gamma_{2}$ is a cycle so $\omega\left(\gamma_{1}\right)-\omega\left(\gamma_{2}\right)$ $=\omega\left(\gamma_{1}-\gamma_{2}\right)$ is an integral multiple of $2 \pi i$ and hence $\exp \left(\omega\left(\gamma_{1}\right)\right)$ $=\exp \left(\omega\left(\gamma_{2}\right)\right)$ so $f$ is well defined. In any simply connected region we can write $\omega$ locally as $d g$ and then clearly in this region we have $f=c e^{g}$ for some constant $c$, and hence $d f / f=d g=\omega$. Since this holds in a neighborhood of each point we have in fact $\omega=d f / f$ so $\omega$ is logarithmically exact. q.e.d.

We now make the assumption that the first Betti number $b_{1}$ of $M$ is finite, i.e. that $H_{1}(M, Z) /$ Torsion is finitely generated. Let $\gamma_{1}, \cdots, \gamma_{b_{1}}$ be a basis for $H_{1}(M, Z) /$ Torsion. By deRham's theorem we can choose closed one-forms $\omega_{1}, \cdots, \omega_{b_{1}}$ on $M$ such that $\omega_{j}\left(\gamma_{k}\right)$ $=\delta_{j k} 2 \pi i$. Then clearly $\Omega\left(\omega_{i}\right) \subseteq 2 \pi i \boldsymbol{Z}$ so by Theorem A each $\omega_{i}$ is a logarithmically exact form, and changing $\omega_{i}$ within its cohomology class if necessary we can assume $\omega_{i}$ is induced. Moreover it follows from Theorem A that a linear combination $\sum_{i=1}^{b_{1}} C_{i} \omega_{i}$ of the $\omega_{i}$ will be logarithmically exact if and only if each $C_{i}$ is integral. Now since a closed one-form has its cohomology class completely determined by its periods over $\gamma_{1}, \cdots, \gamma_{b_{1}}$ it follows that the linear combinations of the $\omega_{i}$ form a linear complement to $B^{1}(M, C)$ in $Z^{1}(M, C)$. Hence:

THEOREM B. If the first Betti number $b_{1}$ of $M$ is finite then the group of logarithmic cohomology classes of $M$ is a free abelian group with $b_{1}$ generators. More precisely there exist $b_{1}$ induced logarithmically exact forms $\omega_{1}, \cdots, \omega_{b_{1}}$ such that their cohomology classes $\left[\omega_{i}\right]$ form an integral basis for $L^{1}(M, \boldsymbol{C}) / B^{1}(M, C)$ and a complex basis for $H^{1}(M, C)$.

The Institute for Advanced Study 